\title{
Actitudes lingüísticas de estudiantes de lenguas de una universidad pública colombiana
}

Primer semestre de 2022 - pp. 153-168 Segunda época

N.

\author{
Linguistic Attitudes \\ of Language Students \\ from a Colombian \\ Public University
}

Atitudes linguísticas de estudantes de línguas de uma universidade pública colombiana

Para citar este artículo

Salazar-Caro, A. (2022). Actitudes lingüísticas de estudiantes de lenguas de una universidad pública colombiana. Folios, (55). https://doi.org/10.17227/folios.55-12217

Profesora de la Universidad Popular del Cesar. Magíster en Investigación Social Interdisciplinaria. Estudiante del doctorado en Lingüística de la Pontificia Universidad Católica de Chile.

Correo: aurarosasalazar@unicesar.edu.co 


\section{Resumen}

En este artículo derivado de investigación se analizan las actitudes lingüísticas de los estudiantes del programa de Licenciatura en Lengua Castellana e Inglés de la Universidad Popular del Cesar. Este estudio cuenta con un enfoque mixto, el cual fue diseñado a partir de un cuestionario de treinta preguntas que se aplicó a los estudiantes matriculados en la asignatura Sociolingüística y Etnolingüística durante el segundo semestre del 2018. Los datos recogidos revelan que los estudiantes prefieren el inglés en ámbitos como la formación profesional y laboral, y el español en escenarios cotidianos y de relaciones interpersonales. Coinciden en que la importancia del inglés radica en que es una lengua que genera oportunidades laborales y académicas. La conclusión principal de este estudio es que los estudiantes tienen una motivación instrumental que sustenta el aprendizaje de inglés en beneficios económicos y laborales que esta lengua reporta.

\section{Palabras clave}

actitudes lingüísticas; español; inglés; aprendizaje; estudiantes

\section{Abstract}

This research article analyzes the linguistic attitudes of the students of the degree in Spanish and English language from the Universidad Popular del Cesar. This study has a mixed focus, designed from a 30-question questionnaire applied to students that were registered in the subject of sociolinguistics and ethnolinguistics during the second semester of 2018. The data collected reveals that the students prefer English in areas such as job and vocational training and Spanish in everyday scenarios and interpersonal relationships. They agree that the importance of English lies in that it is a language that generates job and academic opportunities. The main conclusion of this study is that students have an instrumental motivation that sustains English learning in the economic and job benefits that it reports.

\section{Keywords}

linguistic attitudes; Spanish; English; learning; students

\section{Resumo}

Neste artigo derivado da pesquisa analisam-se as atitudes linguísticas dos alunos do programa de Licenciatura em Língua Castelhana e Inglês da Universidad Popular del Cesar. Este estudo utiliza uma abordagem mista, desenhada por meio de um questionário com 30 questões que foi aplicado aos estudantes inscritos na disciplina de Sociolinguística e Etnolinguística no segundo semestre do ano 2018. Os dados coletados revelam que os alunos preferem fazer uso do inglês nos âmbitos do tipo profissional e de trabalho, e o espanhol nos contextos da cotidianidade e de suas relações interpessoais. Eles concordam que a importância do inglês está em que é uma língua que gera oportunidades laborais e acadêmicas. A principal conclusão deste estudo é que os alunos sentem uma motivação instrumental que ratifica o aprendizado do inglês nos benefícios econômicos e laborais que esta lingua traz consigo.

\section{Palavras chave}

atitudes linguísticas; espanhol; inglês; aprendizagem; alunos 


\section{Introducción}

Las actitudes lingüísticas en contextos educativos constituyen un objeto de investigación reciente que ha facilitado la comprensión de los procesos de aprendizaje de primeras y segundas lenguas. Los estudios en este campo se dividen en dos líneas: los que analizan las actitudes de los estudiantes hacia las lenguas que hablan o estudian (Hernández, 2012; Izquierdo Díaz, 2013; Matos Aldana, 2010; Montoya, 2013); y los que analizan las actitudes de los profesores frente a las lenguas que enseñan o hacia las variedades propias de sus estudiantes (Agudelo et al., 2016; Henríquez y Olate, 2010; Mamani, 2011; Rodríguez, 2018). Los trabajos hechos en la primera línea muestran que la actitud positiva de los estudiantes hacia lenguas extranjeras como el inglés o el francés se sustentan en razones utilitarias, debido a que estas lenguas son consideradas herramientas para lograr beneficios a nivel nacional e internacional.

En este artículo se analizan las actitudes lingüísticas de los estudiantes del programa de Licenciatura en Lengua Castellana e Inglés de la Universidad Popular del Cesar (Valledupar). El análisis se centra en aspectos como las motivaciones y proyecciones de los estudiantes con respecto a las lenguas de especialidad del programa, las creencias que tienen acerca del español y del inglés, la forma como valoran y asumen sus competencias lingüísticas en ambas lenguas, y las preferencias a la hora de elegir una de las dos en ámbitos de cualificación profesional, relaciones interpersonales y actividades cotidianas. Estos aspectos resultan trascendentales en la revisión de las actuales políticas lingüísticas colombianas porque, como plantea Lasagabaster (2004), la mejor forma de instrumentar una política lingüística adecuada y democrática consiste en tener en cuenta las percepciones, creencias y opiniones del mayor número posible de ciudadanos.

El texto se organiza en cinco partes: en la primera se presenta una reflexión teórica en torno al concepto de actitudes lingüísticas, a partir de autores como Trudgill (1993), Moreno Fernández (1998) y Castillo (2006). En la segunda parte se hace un breve recorrido de los estudios de actitudes en contextos educativos y se sitúa el problema de investigación en la Universidad Popular del Cesar. En la tercera parte se presentan los criterios metodológicos que orientaron el proyecto, en la cuarta se incluye el análisis de datos, y en la última se presentan algunas de las conclusiones que surgieron del análisis. 


\section{Acerca del concepto de actitud lingüística}

El estudio de las actitudes lingüísticas resulta fundamental en los contextos de enseñanza de primeras y segundas lenguas, debido al papel central de estas en el proceso de adquisición de una lengua (Janés, 2006; Moreno Fernández, 2007). Desde la psicología social, la actitud se define como una valoración o evaluación de un elemento, evento o hecho de la realidad, fundamentada en creencias, imaginarios sociales, sentimientos, emociones y disposiciones que los sujetos construyen en sus contextos de interacción social y que se relacionan con sus historias de vida.

En el ámbito lingüístico, las actitudes se relacionan con las valoraciones positivas o negativas que tienen los hablantes con respecto a las lenguas. Moreno Fernández define la actitud lingüística como

Una manifestación de la acción social de los individuos distinguida por centrarse y referirse específicamente tanto a la lengua como al uso que de ella se hace en sociedad, y al hablar de lengua incluimos cualquier tipo de variedades lingüísticas: actitudes hacia estilos diferentes, sociolectos diferentes, dialectos diferentes o lenguas naturales diferentes. (1998, p. 179)

En este sentido, la actitud lingüística consiste en la evaluación que hacen los hablantes de la lengua o del uso que le da determinada comunidad, a partir de las creencias y el grado de vinculación social, cultural y emotiva que han establecido con las lenguas o con sus usuarios. Castillo define las actitudes como "manifestaciones valorativas que proyectan la percepción que se tiene de las personas de grupos diferentes que hablan determinada lengua" (2006, p. 286). En consonancia con esta definición, el autor plantea que en los estudios de las actitudes lingüísticas hay que considerar lo que dice la gente acerca de la lengua y acerca de sus funciones y usos sociales, porque lo que los hablantes expresan refleja la forma como valoran y perciben a los demás. Al respecto, Falcón y Mamani (2017) consideran que las actitudes lingüísticas involucran un juicio no solo frente a la lengua, sino frente a los hablantes y sus comportamientos lingüísticos. Por tanto, las actitudes tienen que ver con el posicionamiento social que se atribuya a los hablantes.

De acuerdo con Castillo (2007), las actitudes lingüísticas pueden ser favorables o desfavorables y van a estar determinadas por los intereses sociales $y$ comunicativos de los hablantes, y por los contextos de uso y el grado de prestigio del que goce la lengua en escenarios interculturales. En este sentido, se podría afirmar que la valoración lingüística que hacen los hablantes se relaciona con el grado de prestigio lingüístico de la lengua y de sus usuarios. De hecho, Trudgill (1983) plantea que los juicios de valor sobre las lenguas se sustentan en el estatus social de sus hablantes; y González (2008) señala que las actitudes tienen implícitos conceptos como la conciencia sociolingüística y el prestigio, así como nociones socioculturales, económicas y políticas que trascienden al sistema lingüístico.

Desde el punto de vista teórico y metodológico, los estudios de las actitudes se han hecho desde dos enfoques: conductista y mentalista. En el primero, las actitudes se encuentran en las respuestas de la gente ante una lengua o situación determinada y se analizan a partir de la observación directa. En el segundo, la actitud se asume como un estado interno o una disposición frente a una lengua, por lo que se suelen emplear entrevistas o técnicas de pares ocultos que permitan acceder a las actitudes por medio de la inferencia. Ambos enfoques presentan limitaciones metodológicas: en el mentalista la actitud puede predecirse, pero no puede comprobarse a través de la observación y la experimentación. ${ }^{1}$ En el enfoque conductista las actitudes se pueden constatar de forma directa a través de la observación, pero no son predecibles (Alcázar y Atencia, 2017; Blas Arroyo, 1999; Castillo, 2006; González, 2008).

1 Respecto a esta limitación, Blas Arroyo (1999) plantea que el hecho de que los estados mentales no puedan ser observados de forma directa, y deban ser inferidos a partir las confesiones o los discursos de los hablantes, afecta en algunas ocasiones la validez de los datos. A pesar de lo anterior, la mayoría de los investigadores de las actitudes lingüísticas se adhieren al enfoque mentalista (Blas Arroyo, 1999), por lo que se considera que es el que cuenta con mayor tradición y respaldo investigativo. 
En sociolingüística, los estudios se han hecho generalmente desde el enfoque mentalista con el modelo multicomponencial. Gómez (1998) y Hernández-Campoy (2004) plantean que este modelo se integra desde un componente cognitivo formado por conocimientos, pensamientos y creencias de hablantes sobre una lengua; un componente emotivo del que forman parte todos los sentimientos de estima o rechazo que se generan con respecto a una lengua o variedad; $y$ un elemento conativo relacionado con la predisposición o disponibilidad para actuar en un contexto sociolingüístico determinado. El cuestionario diseñado para este estudio se hizo considerando cada uno de esos componentes y los elementos que abarcan (véase "Metodología").

En este artículo se analizan las actitudes lingüísticas en un contexto de enseñanza de español y de inglés, siendo la primera la lengua materna y la segunda la lengua extranjera. Se decidió estudiar estas dos lenguas, teniendo en cuenta que diversos autores coinciden en reconocer el importante papel de la lengua materna en el aprendizaje de una segunda lengua (Klein, 1986; Buck, 1994; García y Senovilla, 1998; Benítez 2004-2005), y que parte de esa importancia se relaciona con los procesos de interferencia de la L1 en la L2 que algunos autores interpretan como fenómenos negativos que promueven errores y retardan el aprendizaje (Benítez, 2004-2005; Malinowsky, 2000). Cabe aclarar que la investigadora de este estudio difiere con estos autores, debido a que considera que la primera lengua constituye el soporte para la adquisición de una lengua extranjera, y que los fenómenos de interferencia son naturales y necesarios para lograr transitar de una L1 a una L2.

Sin embargo, la idea de que la interferencia del español retrasa el aprendizaje del inglés está generalizada y podría afectar las actitudes de los estudiantes hacia su lengua materna. A lo anterior se suma el hecho de que se suele desvalorizar al español debido a que el inglés se considera la clave del éxito por el lugar que ocupa en los contextos académicos y económicos (Criado, 2004). De acuerdo con Cantos y Sánchez (2011), aunque ambas lenguas están equiparadas en número de hablantes, en el contexto mundial "el inglés ocupa una preeminencia y liderazgo indiscutibles" (p. 18). Estos fenómenos sociales y lingüísticos señalan la necesidad de analizar las actitudes hacia las lenguas extranjeras y propia en un escenario de formación profesional donde el español y el inglés se deberían reconocer por su valor social y cultural y el lugar que ocupan en el sistema educativo colombiano.

Este estudio tiene mayor impacto si se vincula con las políticas educativas y lingüísticas actuales. La propuesta del Ministerio de Educación Nacional (2014) de fortalecer las competencias de los estudiantes en inglés mediante iniciativas como el acompañamiento y la formación docente, la motivación a los estudiantes y el aumento de número de horas de inglés debe estar sustentada en el conocimiento de las actitudes de docentes y estudiantes hacia el español y el inglés. Ese es un factor fundamental al que no se le ha dado la importancia que merece en los programas nacionales de bilingüismo; la mayoría de estos promueve entre la comunidad una motivación instrumental sustentada en la idea de que en Colombia se debe aprender inglés para lograr oportunidades laborales, acceso a estudios en el exterior y aumento de ingresos, como plantea el Programa Nacional de Inglés 2015-2025.

\section{Estudios sobre las actitudes lingüísticas en contexto de formación}

En la línea de las investigaciones sobre actitudes lingüísticas de los estudiantes hacia la lengua que hablan o acerca de las lenguas extranjeras que estudian ${ }^{2}$ hay que resaltar varios trabajos internacionales y nacionales. En el País Vasco, Lasagabaster (2004) analizó las actitudes lingüísticas de los estudiantes

2 En esta línea hay tres focos: 1) las actitudes de estudiantes con distintos grados de bilingüismo hacia la lengua minoritaria y hacia la lengua dominante (Canché Teh, 2014; Huguet y Llurda, 2001; Lasagabaster, 2003), 2) actitudes de estudiantes hispanohablantes hacia la lengua extranjera que están aprendiendo en la escuela o la universidad (Matos Aldana, 2010) o hacia su propia lengua como resultado del aprendizaje de la LE (Izquierdo Díaz, 2013) y 3) actitudes de estudiantes de otra lengua materna hacia el español como lengua extranjera (Masuda, 2014). 
hacia el inglés. Organizó trece grupos de discusión con 41 estudiantes de primer curso de lengua inglesa de la Facultad de Filología y Geografía e Historia de la Universidad del País Vasco-Euskal Herriko Unibertsitatea. Los resultados muestran que entre el alumnado existe una clara conciencia sobre el carácter útil y la potencialidad comunicativa de la lengua inglesa en la vida diaria, ya sea para comunicarse con los extranjeros o para chatear, teniendo en cuenta que están en permanente interacción con estudiantes extranjeros. El autor concluye que la actitud positiva de los estudiantes hacia el inglés se sustenta en una perspectiva instrumental, producto de la situación actual del mercado laboral; y en argumentos sociológicos, económicos y políticos centrados en la utilidad y rentabilidad de la lengua inglesa en los escenarios académicos, profesionales y laborales, en los que goza de un alto grado de prestigio.

En México, Muñoz (2019) desarrolló un estudio con el objetivo de analizar las actitudes y creencias lingüísticas en relación con la enseñanza de la lengua materna en estudiantes universitarios. La autora planteó interrogantes como ¿qué es lo que piensa un estudiante de licenciatura acerca de su propia lengua?, ¿por qué se cree que dominar una lengua extranjera abrirá las puertas del éxito?, ¿cómo se justifica que se considere una lengua mejor que otra?, ¿ debe el individuo enfrentar contradicciones internas acerca de su lengua materna, odiarla y amarla al mismo tiempo? En términos metodológicos, aplicó un cuestionario de tres preguntas a noventa estudiantes de Medicina Humana, Derecho e Ingeniería Civil de la Universidad Autónoma de Zacatecas.

Los datos revelan que la mayoría (60\%) de los estudiantes quisiera que el inglés fuera su lengua materna, porque considera que es el idioma universal y brinda más oportunidades laborales y académicas que el español. El $60 \%$ considera que no habla correctamente el español porque emplea variaciones dialectales y tiene dificultades con el dominio del sistema ortográfico. El $80 \%$ opina que sería bueno que en las escuelas las clases de ciencias se impartieran en inglés porque el conocimiento científico se desarrolla más en países de habla inglesa. La autora argumenta que las actitudes favorables de los estudiantes hacia el inglés se sustentan en la concepción del bienestar económico como indicador del éxito profesional y la convicción de que la homogeneización cultural y científica, que implica la subordinación del español frente al inglés, es conveniente porque facilitará el progreso y la entrada al primer mundo.

En Bogotá, Dávila (2012) analizó el capital lingüístico y la desigualdad de oportunidades en los procesos de enseñanza de inglés en dos colegios oficiales de Bogotá. La autora resalta que los principales objeciones que han surgido en Colombia en materia de políticas sobre bilingüismo tienen que ver con la invisibilización de los docentes como actores fundamentales en la implementación de estrategias de adquisición de lengua extranjera y el desconocimiento de las múltiples variables (sociales, económicas, educativas, institucionales y actitudinales) presentes en la sociedad colombiana que no se tomaron en cuenta a la hora de asumir el Marco Común Europeo (MCE) como referencia para la enseñanza y el aprendizaje del inglés. Plantea que uno de los aspectos críticos en este campo es el papel del docente y sus actitudes hacia su quehacer profesional.

En cuanto al nivel de motivación de los estudiantes hacia el inglés, la autora identificó que en las instituciones estudiadas el inglés ocupa el segundo lugar en la lista de asignaturas consideradas importantes. Sin embargo, descubre que quienes le conceden mayor importancia no son quienes logran mejores resultados, por lo que concluye que la motivación y el gusto no son lo único que lleva a que los estudiantes se dediquen al aprendizaje de una lengua. Señala que la mayoría de los estudiantes de los colegios analizados manifiesta que les gusta el inglés como idioma en general y la clase de inglés en particular, y reconoce la importancia del conocimiento de esta lengua en el logro de mejores oportunidades educativas, laborales y culturales. Sin embargo, concluye que el imaginario frente al 
bilingüismo español-inglés se fundamenta en que la población estudiantil de estas instituciones considera que no necesita y/o que no puede aprender inglés. La mayoría reconoce que el inglés no es útil ni significativo debido a que en sus contextos cotidianos no hay ninguna actividad que requiera del inglés para su desarrollo, excepto las clases de este idioma.

En ese mismo grupo se ubica el estudio "La construcción de las actitudes lingüísticas: exploración en dos colegios bilingües bogotanos", desarrollada por Montoya (2013). La autora se propone, por un lado, caracterizar las actitudes lingüísticas de los estudiantes de undécimo grado de dos colegios bilingües español-inglés, uno privado y otro público, hacia su lengua materna y hacia la extranjera. Y por otro, determinar los factores que inciden en esas actitudes lingüísticas. Para ello, diseñó y aplicó cuestionarios a estudiantes, docentes y padres de familia. En la presente investigación se tomó como base el cuestionario aplicado a estudiantes.

De este estudio resultó que en general estudiantes, padres de familia, docentes y administrativos de ambas instituciones educativas tienen actitudes positivas hacia el inglés y el español, y que existen diferencias y similitudes en el tipo de apreciaciones que tienen sobre los dos idiomas. Entre los participantes el inglés es visto como una herramienta para lograr beneficios prácticos a nivel nacional e internacional, y el español se considera principalmente como el medio de comunicación cotidiana. Existe una tensión entre los componentes de las actitudes lingüísticas ya que a pesar de que opinan que el inglés es importante, los estudiantes no tienen la suficiente motivación para mejorar su competencia lingüística.

Como puede notarse, la mayoría de estas investigaciones analizan las actitudes de los estudiantes hacia el inglés y el español, y concuerdan en que las actitudes favorables hacia el inglés se sustentan en su utilidad para lograr beneficios económicos, académicos y laborales. Montoya (2013) examina la incidencia de las políticas lingüísticas en las actitudes de los estudiantes y señala la necesidad de hacer investigaciones que analicen en profundidad las actitudes lingüísticas y su incidencia en la educación bilingüe colombiana. El presente estudio responde a esa necesidad y tiene una particularidad y es que estudia las actitudes de quienes se están formando como docentes de español y de inglés. Los informantes de la investigación tendrán en unos años la responsabilidad de formar a las nuevas generaciones; por tanto, es fundamental conocer sus actitudes hacia las lenguas extranjeras para plantear una discusión en torno al lugar de estas en políticas lingüísticas actuales como la Ley 1651 del 2013 y el Programa Nacional de Inglés 2015-2025.

\section{Metodología}

Este es un estudio con enfoque mixto teniendo en cuenta que complementa elementos cualitativos y cuantitativos. Hernández-Campoy (2004) plantea que en los trabajos sobre actitudes lingüísticas resulta útil complementar los dos enfoques. Se sigue el método directo para el análisis de las actitudes, que consiste en solicitar de forma explícita la opinión de los hablantes sobre una lengua (Castillo, 2006). Se acoge el modelo mentalista multicomponencial, ampliamente sustentado por autores como Gómez (1998, 2002) y Hernández-Campoy (2004) (véase el apartado "Estudios sobre las actitudes lingüísticas en contexto de formación”). El trabajo fue realizado con treinta estudiantes de sexto y séptimo semestre de la Licenciatura en Lengua Castellana e Inglés que matricularon la asignatura Sociolingüística y Etnolingüística durante el segundo semestre del año 2018 , periodo en el que se desarrolló el proyecto. ${ }^{3}$ La recolección de los datos se hizo a través de un cuestionario virtual de treinta preguntas ${ }^{4}$ diseñado a partir de los tres componentes del modelo

3 Los datos se recolectaron durante el primer corte evaluativo del semestre, cuando todavía no se había abordado el capítulo de actitudes lingüísticas, para lograr un acercamiento a las actitudes reales de los estudiantes y, a partir de estos resultados, introducir la reflexión teórica sobre conceptos como la evaluación sociolingüística y el prestigio lingüístico. La investigadora quiso aprovechar los cursos que tenía asignados ese semestre para desarrollar el proyecto y promover el enfoque investigativo que se plantea en el diseño curricular de la asignatura.

4 Por cuestiones de extensión, aquí solo se analizan algunas de las preguntas más relevantes. 
mentalista (cognitivo, emotivo y conativo), explicado por Gómez (1998; 2002).

El instrumento aplicado contiene preguntas abiertas y cerradas, lo que permitió categorizar los datos desde los discursos de los estudiantes. Blas Arroyo (1999) plantea que las preguntas abiertas permiten que el informante tenga una mayor libertad de expresión. De las ventajas de los cuestionarios señaladas por Hernández-Campoy y Almeida (2005) se evidenciaron las siguientes: economía de tiempo y recursos; ausencia física del investigador y posibilidad de incluir preguntas abiertas y cerradas. Algunas de las preguntas fueron adaptadas de los trabajos de Matos Aldana (2010) y Montoya (2013). En el componente cognitivo aparecen preguntas asociadas a la forma como los estudiantes conciben o califican las lenguas objeto de estudio, así como a la valoración de su rendimiento en ambas lenguas en términos del programa al que están adscritos. Las preguntas que tienen que ver con el rendimiento se hicieron con el ánimo de contrastar las respuestas de esa autoevaluación con los informes de eficiencia académica de las asignaturas, y analizar si existe relación entre el rendimiento y las actitudes lingüísticas expresadas. ${ }^{5}$

El componente emotivo está centrado en el gusto o preferencia de los estudiantes por cada lengua y las motivaciones que tienen para continuar en el programa. El aspecto motivacional se incluye aquí en la medida en que se considera que las actitudes se correlacionan con la motivación y los resultados logrados con respecto al aprendizaje de una lengua extranjera (Minera, 2010). Se incluyeron preguntas como ¿le gusta estudiar inglés?, ¿usted quiere ser docente de español, o de inglés?, ¿por qué?, ¿qué es lo que más le gusta del inglés?, ¿cuál es su principal motivación para continuar en el programa de Licenciatura en Lengua Castellana e Inglés?

En el componente conativo están aquellas cuestiones relacionadas con los usos del inglés en diferentes contextos comunicativos, la preferencia de una u otra lengua en ámbitos laborales o de formación profesional y las asignaturas a las que

5 Estos objetivos se cumplirán en un próximo estudio. los estudiantes les dan mayor importancia. Estas preguntas se centraron en situaciones del tipo: $\mathrm{Si}$ hubiera tenido la posibilidad de elegir entre estudiar español y estudiar inglés, ¿cuál hubiera elegido? En redes sociales, ¿usa la mayoría de veces el español o el inglés? ¿A qué materias les da mayor prioridad en el estudio: a las del español, o a las del inglés?

\section{Análisis de datos}

Los resultados están organizados a partir de dos temas: la importancia que los estudiantes le atribuyen al aprendizaje del inglés y el español; y las preferencias en los campos de formación profesional, actividades cotidianas y relaciones interpersonales. Estas dos temáticas permitieron fusionar preguntas de naturaleza cognitiva (¿por qué considera que es importante aprender inglés?, ¿por qué cree que es importante estudiar español?); emotiva (¿usted quiere ser docente de español, o de inglés?, ¿por qué?, ¿qué le desagrada del inglés?) y conativa (al terminar la licenciatura, ¿se especializará en el estudio del español, o del inglés?; si lo pusieran a elegir entre ser docente de español y de inglés, ¿cuál escogería?; para ver televisión, leer, relacionarse con sus amigos y escribir, ¿prefiere el español, o el inglés?).

Como puede notarse, en el análisis se incluyeron preguntas abiertas y cerradas. En las cerradas se cuantificaron los resultados, se sacaron los porcentajes y se representaron a través de gráficos de Excel. Para las preguntas abiertas se siguieron algunas de las pautas planteadas en el proyecto Linguistic Identity and Attitudes in Spanish-Speaking Latin America (LIAS), por lo que se identificaron una serie de categorías para cada grupo de preguntas, a partir de cuadros de Excel para cada respuesta, que generaron grupos de respuestas, tendencias y resultados relevantes (Bernal et al., 2014).

\section{La importancia de aprender inglés y español}

En esta categoría, los estudiantes coinciden en que la importancia de aprender inglés radica en que es una lengua que abre oportunidades laborales y académicas, permite entrar en diálogo con otras 
culturas y países. Los informantes justifican que es importante aprender inglés:

abre muchas puertas y da más oportunidades de conocer una nueva cultura. (INF03)

nos brinda más oportunidades en el campo laboral. (INF01)

es necesario aprender un nuevo idioma, si se puede considerar la idea de irse fuera del país. (INF05)

en un mundo donde hay desarrollo y avances cada momento es necesario ir creciendo profesionalmente, además de lograr conseguir un buen trabajo. (INF06)

te ayuda a conocer países y sus culturas; también te brinda la oportunidad de tener un mejor trabajo. (INF08)

es una necesidad para poder adquirir mejores oportunidades de estudio y trabajo. (INF011).

ya que es el idioma esparcido en diferentes países del mundo y al aprenderlo puedes viajar. (INF014)

abre las puertas hacia un nuevo mundo (INF016)

el inglés es denominado el idioma universal, si sabes hablar inglés te puedes desenvolver fácilmente en cualquier lugar del mundo (INF017)

puedo conocer otra cultura y lograr mejores condiciones de trabajo. (INF019)

Como puede verse, la creencia recurrente es que el manejo del inglés abre las posibilidades para mejorar las condiciones laborales dentro o fuera del país. Hay quienes ven su aprendizaje desde la posibilidad de encuentro con culturas diversas. En los discursos de los estudiantes está arraigado el deseo de salir del país a conectarse con otros espacios o buscar oportunidades de profesionalización. Estos resultados coinciden con los encontrados por Lasagabaster (2004), según los cuales las actitudes favorables de los estudiantes universitarios vascos hacia el inglés obedecen a una elección de orden económico fundamentada en su rentabilidad práctica en el mundo laboral, profesional o comercial. Según el autor, "estas actitudes denotan una clara percepción del grado de prestigio del inglés por su utilidad social inmediata" (p. 100).

En estos resultados se evidencia que hay una pugna entre lo que los estudiantes creen acerca del inglés y la manera como se vinculan emocionalmente con esta lengua. Está claro que la ven desde las ventajas laborales y económicas que ofrece; tienen lo que Janés (2006) denomina una motivación instrumental, que es aquella que impulsa a los sujetos a aprender una segunda lengua con propósitos utilitarios y prácticos, como encontrar un mejor trabajo, obtener reconocimiento social o conseguir ventajas económicas. Sin embargo, en este caso estas ventajas no promueven el gusto o la pasión por aprender la lengua. En el estudio de Lasagabaster (2004) se encontró que los estudiantes vascos también carecen de argumentos afectivos que sustenten una actitud favorable hacia el aprendizaje del inglés.

En la pregunta relacionada con aquellos aspectos que les resultan desagradables del inglés, aunque el $21 \%$ reconoce que no le desagrada nada, la mayoría señala que los aspectos que más les disgustan están relacionados con el habla y las formas gramaticales, que son además los que fundamentan el aprendizaje de esta lengua (véase la figura 1). Algunos estudiantes llegan incluso a catalogar como caprichosa la gramática inglesa, debido a que les resulta difícil aplicar una lógica para comprender estas reglas.

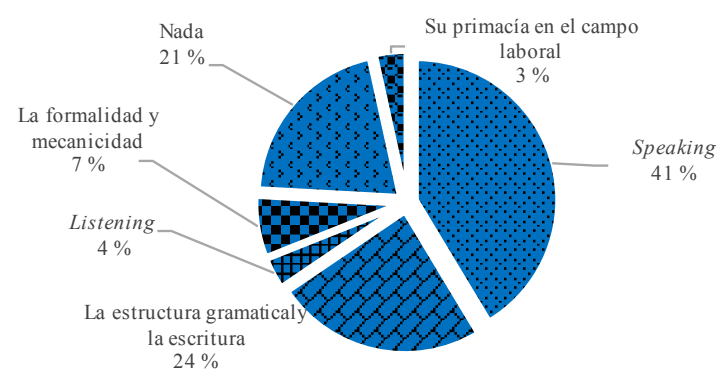

Figura 1. ¿Qué le desagrada del inglés?

Fuente: elaboración propia 
Este es un caso en el que los componentes de las actitudes se encuentran en conflicto: por un lado, hay una motivación instrumental asociada a la necesidad de aprender inglés para lograr un mejor posicionamiento laboral y social; y por el otro está la falta de vinculación emocional generada por aquellos aspectos de esta lengua que no les resultan agradables y que probablemente influyan de forma negativa en las actitudes de los estudiantes hacia su aprendizaje. Dávila (2012) señala que la motivación y el gusto no son lo único que lleva a que los estudiantes se dediquen al aprendizaje de una lengua, que puede haber un alto nivel de compromiso con la formación académica, independientemente del agrado. Sin embargo, la investigadora de este estudio coincide con Montoya (2013) en que la motivación constituye un aspecto fundamental en el mejoramiento de las competencias comunicativas en una lengua extranjera.

Por otro lado, cuando se les pregunta acerca de la importancia de aprender español, presentan fundamentalmente dos argumentos: primero, porque es su lengua materna, la que los identifica. Segundo, porque es una lengua reconocida a nivel mundial. En sus relatos señalan:

para conocer más sobre nuestra lengua materna, sus usos y compartir todo esto con los demás. (INF01)

es un idioma que su importancia trasciende, no disminuye en la sociedad y sobre todo, que es mi lengua materna y debo amarla y dedicarle tiempo para aprender cosas nuevas cada día. (INF04)

ante todo, considero que es importante conocer la lengua materna, además de expresarse de forma adecuada según sea el contexto y utilizar las demás habilidades del español. (INF06)

es necesario tener conocimientos claros de la lengua que hablamos. (INF07)

para conocer más sobre nuestra lengua. (INF08)

antes de aprender otra lengua, debemos aprender la nuestra. (INF09) primeramente, porque es un idioma difícil de aprender y porque es el segundo o tercero de hablar mundialmente. (INF011)

es la forma de comprender y de leer a las personas, además como lengua madre nos ayuda a saber más sobre nuestras raíces. (INF014)

en nuestro caso es la lengua materna y es importante saber todo sobre nuestro idioma. (INF014)

es importante tener conocimiento de nuestra propia lengua, conocimiento tanto histórico como gramático. (INF021)

es mi lengua madre y además es uno de los idiomas más importantes en el mundo. (INF024)

para apropiarnos de ella y nuestra identidad. (INF025)

al ser la segunda lengua más hablada en el mundo, considero que es indispensable aprenderlo. (INF026)

En estos discursos se observa un mayor grado de vinculación emocional por parte de los estudiantes en relación con su primera lengua. Unos dicen que hay que amarla y conocerla porque forma parte de sus raíces, de su identidad, de su sentido de pertenencia a la comunidad hispanohablante. Esta postura es coherente con lo que propone Lopreto (2018) cuando señala que

La lengua primera es esa que lo integra a la cultura de origen y de pertenencia: a su familia, su nación, su poesía, sus derechos, sus aprendizajes. La primera lengua es la de los afectos. [...] Se adquiere junto a los primeros afectos y es además el instrumento con el que se inicia el conocimiento del mundo, directamente vinculada a un sentimiento de identidad y de pertenencia. (pp. 324-325)

Para otros estudiantes, la importancia del español radica en el lugar y el prestigio que ha logrado en tiempos de globalización. López Morales (2010) considera que el prestigio del español se debe al número de hablantes, la relativa homogeneidad 
lingüística de la que goza y el protagonismo indiscutible que ostenta en universidades, bibliotecas, archivos y medios de comunicación masivos. Sin embargo, al contrastar la importancia que dan los estudiantes al español y al inglés, las diferencias son claras: mientras el inglés se relaciona con el estatus económico, comercial y laboral que ha adquirido, el español es valorado principalmente porque es su primera lengua, con la creen que deberían sentirse identificados; y no porque represente alguna ventaja en términos de profesionalización o de posicionamiento social. Es evidente aquí la preeminencia del inglés sobre el español a la que se refiere Cantos y Sánchez (2011), cuando afirman que, en el contexto mundial, "el inglés ocupa una preeminencia y liderazgo indiscutibles" (p. 18).

\section{Preferencias lingüísticas en el entretenimiento y la formación}

Respecto a la pregunta "Si hubiese tenido la posibilidad de elegir entre estudiar español y estudiar inglés, ¿cuál hubiera elegido?" se observa en la figura 2 que el $63 \%$ de los estudiantes elegiría el inglés en lugar del español. Estos resultados probablemente se relacionan con las ventajas laborales que ofrece el aprendizaje de esta lengua extranjera, un hecho al que se aludió en la pregunta anterior. La mayoría de los estudiantes habría preferido estudiar inglés.

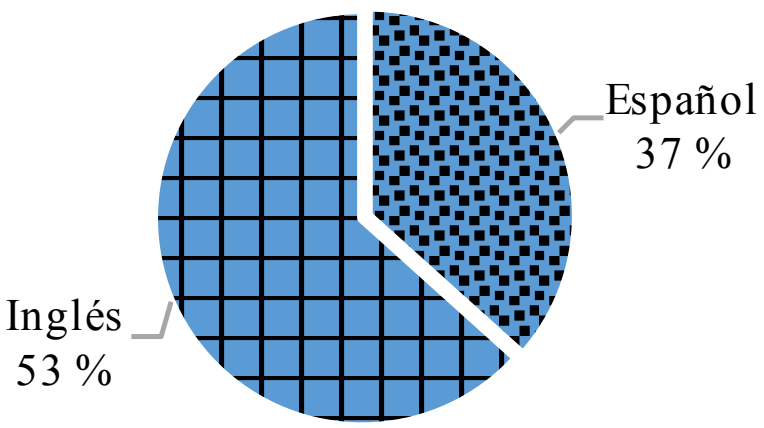

Figura 2. Si hubiera tenido la posibilidad de elegir entre estudiar español y estudiar inglés, ¿cuál lengua habría elegido?

Fuente: elaboración propia

También se les preguntó por la lengua de preferencia para especializarse, y como se observa en la figura 3, la mayoría prefiere el inglés. A partir de estos resultados, se podría decir que en los campos educativos y laborales, prefieren el inglés. Estos resultados podrían contrastarse con los de la siguiente pregunta, donde queda claro que para las relaciones sociales y la diversión, los estudiantes prefieren su lengua materna. Se reafirma la permanencia de una motivación instrumental, centrada en las ventajas económicas y académicas. Estos datos coinciden con los hallados por Dávila (2012) y Montoya (2013) en Bogotá, donde se evidenció que ya desde la escuela el inglés es visto como una herramienta para lograr beneficios prácticos a nivel nacional e internacional, en términos de mejores oportunidades educativas y laborales.

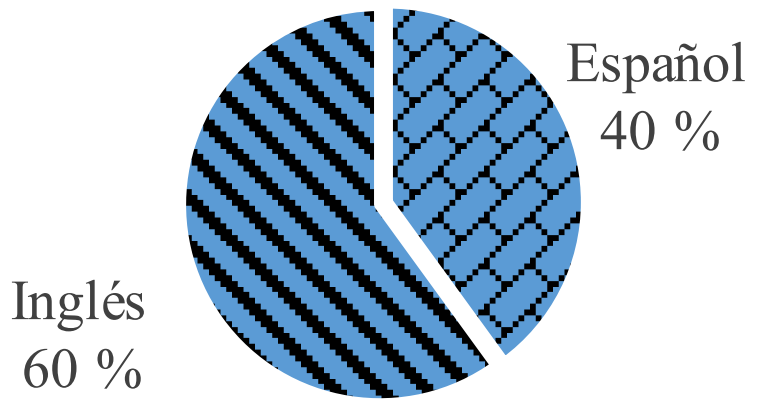

Figura 3. ¿Le gustaría especializarse en el estudio del español o del inglés?

Fuente: elaboración propia

Como muestra la figura 4, el $80 \%$ manifiesta preferir su lengua para hacer actividades como ver televisión, leer, escribir y relacionarse con los amigos; y solo el $20 \%$ prefiere usar el inglés en este tipo de actividades. Este hecho podría revelar el grado de vinculación de los estudiantes con la lengua extranjera y reafirma lo señalado en anteriores preguntas: ven esta lengua como una posibilidad de ascenso social y laboral, pero no la sienten como un sistema lingüístico predilecto. Esta elección plantea la necesidad que en la Universidad Popular del Cesar, en los programas de lenguas, se promueva el uso del inglés en contexto de comunicación diversos, que estimulen el gusto por la lengua. 
Universidad Pedagógica Nacional

Facultad de Humanidades

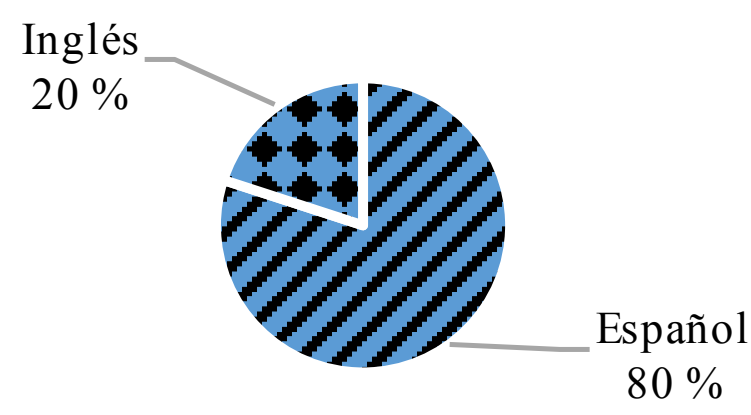

Figura 4. Para ver televisión, leer, escribir y relacionarse con amigos, ¿prefiere el español o el inglés?

Fuente: elaboración propia

Estos resultados se relacionan con lo que señala Requejo (1995, citado por Lopreto, 2018) cuando resalta la vinculación de la lengua materna con la comunidad de origen y plantea que las producciones lingüísticas de la vida cotidiana en el contexto familiar y social, ligadas al espacio y el entorno ambiental, actúan sobre los modos de entender las relaciones, los vínculos, la identidad propia y la vida misma. Por tanto, se comprende que el español sea la lengua predilecta en ámbitos de mayor intimidad o vinculación emocional. Sin embargo, habría que aclarar aquí que los estudiantes prefieren el inglés a la hora de especializarse o cualificarse por las ventajas económicas que podría representar el manejo de esta lengua.

Finalmente, hubo una pregunta relacionada con las preferencias de los estudiantes en términos laborales. El 67 \% elegiría ser docente de inglés, sigue primando el valor utilitario o instrumental que se le da a la lengua y que motiva su aprendizaje. En las figuras 4 y 5 se observa que hay dos ámbitos de elección: para el campo laboral y académico; y para el campo de las actividades cotidianas y de las relacionales interpersonales. En el primer campo se prefiere el inglés.

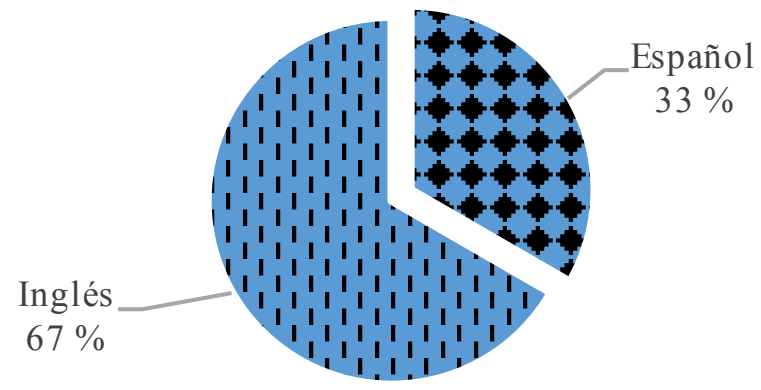

Figura 5. Si tuviera que elegir entre ser docente de español o de inglés, ¿cuál escogería?

Fuente: elaboración propia

En el segundo campo, algunos estudiantes expresan que ven películas en inglés e interactúan con sus compañeros como una estrategia para facilitar su proceso de adquisición de la lengua. Sin embargo, queda claro que en sus espacios de ocio, familiaridad y amistad, prefieren usar su lengua materna. Dávila (2012) encontró que en Bogotá los estudiantes de los colegios oficiales no reconocen la utilidad de aprender inglés, debido a que en sus contextos cotidianos no participan en actividades que impliquen el uso de esta lengua. Por tanto, los resultados de estos estudios ponen en evidencia la necesidad de que en las escuelas y universidades del país se promuevan usos del inglés en escenarios distintos al aula, a fin de que se trascienda la motivación instrumental que caracteriza actualmente las actitudes de los estudiantes de los colegios oficiales y de los programas de formación profesional.

\section{Conclusiones}

Los estudiantes creen que el inglés abre las posibilidades para mejorar las condiciones laborales. Prefieren esta lengua a la hora de cualificarse profesional y laboralmente porque consideran que representa mayores ventajas a nivel nacional e internacional. Hay entre los estudiantes una motivación instrumental que sustenta el aprendizaje de inglés en argumentos económicos. Es claro que muchas de estas actitudes coinciden con la de los estudiantes de Medicina, Derecho e Ingeniería Civil de Zacatecas 
(Muñoz, 2019); de Filología y Geografía e Historia de la Universidad del País Vasco (Lasagabaster, 2004); y de algunos colegios oficiales de Bogotá (Dávila, 2012; Montoya, 2013).

El español, por su parte, es valorado por los estudiantes porque es su primera lengua, con la que creen deberían sentirse identificados. De hecho, expresan una vinculación emocional con su lengua en la medida en que la prefieren en sus relaciones sociales más íntimas y en actividades de entretenimiento. Sin embargo, no reconocen la utilidad de estudiarla en sus proyecciones de cualificación profesional o laboral. Este hallazgo motiva una discusión en torno a las implicaciones culturales y sociales del desarrollo de competencias comunicativas en español en todos los niveles educativos. En Colombia, el estudio de la lengua materna está regulado desde las políticas educativas que buscan promover su valoración como medio de expresión literaria en el país y en el mundo (Congreso de la República, 1994).

Los resultados de este estudio tienen implicaciones nacionales e institucionales. En términos nacionales, deben contemplarse en el marco del Programa Nacional de Inglés 2015-2025, teniendo en cuenta que las actitudes lingüísticas de la población estudiantil constituyen "un factor fundamental a la hora de poner en práctica políticas lingüísticas acertadas" (Lasagabaster, 2004, p. 93). En este caso, las actitudes de los estudiantes demuestran la necesidad de promover una motivación integradora fundamentada en la formación intercultural y el reconocimiento del valor del inglés y del español. A las políticas de bilingüismo del país se les reclama el desconocimiento de las actitudes lingüísticas de la ciudadanía y del papel de los docentes como actores fundamentales en la implementación de estrategias de adquisición de lenguas extranjeras (Dávila, 2012). Por tanto, estos resultados se podrían tomar en cuenta para la evaluación del plan de bilingüismo vigente y para la elaboración de una propuesta de mejora que vincule el aprendizaje del inglés al desarrollo social y cultural del país y que trascienda la motivación instrumental o económica.
En términos institucionales, es preciso discutir aspectos como el papel de las actitudes lingüísticas de los estudiantes en la consolidación de la política lingüística de la Universidad y el tipo de motivación que se está promoviendo para el aprendizaje del inglés y del español. El programa de Licenciatura en Lengua Castellana e Inglés se encuentra en un proceso de transformación que ha posibilitado los ajustes curriculares necesarios para lograr altos niveles de calidad y responder a las exigencias del contexto regional, nacional y global. Este escenario constituye el espacio propicio para formular propuestas que se puedan vincular a las políticas lingüísticas nacionales.

Hay que concluir señalando la necesidad de adelantar en la Universidad Popular del Cesar, en el Caribe y en Colombia estudios que permitan: 1) conocer las reales motivaciones de docentes $y$ estudiantes de lenguas; 2) comprender el papel de las actitudes en el aprendizaje de lenguas extranjeras; 3) reflexionar en torno a cómo desde las políticas lingüísticas y desde los programas de formación básica y profesional se podría trascender la motivación instrumental, y promover el uso del inglés en contexto de comunicación variados, y 4) discutir sobre las implicaciones culturales y sociales que tiene el desarrollo de las competencias comunicativas en lengua materna en los procesos de formación integral.

\section{Referencias}

Agudelo, C., Pasuy, G. Escobar, E. y Ramírez, O. (2016). Actitudes lingüísticas de los profesores de lenguas de la Universidad de Caldas respecto al voseo. Revista de Investigaciones UCM, 16(27), 16-28. http://www. revistas.ucm.edu.co/ojs/index.php/revista/article/ view/57/pdf

Alcázar, A. y Atencia, F. (2017). El habla de Munera (Albacete): Actitudes y percepciones. En A. Bravo, Materiales para el estudio de las actitudes lingüísticas (y II): Las variedades dialectales, el español correcto. Tonos Digitales, 33, 11-57. https://www. um.es/tonosdigital/znum33/secciones/corpora-materiales_para_el_estudio_de_las_actitudes_linguisticas_(y_ii)_definitivo_publicacion.pdf 
Benítez, C. (2004-2005). Estudios de la interferencia del español en el aprendizaje de una lengua extranjera: El caso de los trabajos fin de carrera [tesis doctoral, Universidad Nacional de Educación a Distancia]. http://e-spacio.uned.es/fez/eserv/tesisuned:Filologia-Cdbenitez/BENITEZ_CORREA_CarmenDelia_ Tesis.pdf

Bernal, J., Munévar, A. y Barajas, C. (2014). Actitudes lingüísticas en Colombia. En A. B. Chiquito y M. A. Quesada Pacheco (eds.), Actitudes lingüísticas de los hispanohablantes hacia el idioma español y sus variantes, volumen 5 (pp. 180-245). Bergen Language and Linguistic Studies (BeLLS). http:// dx.doi.org/10.15845/bells.v5i0.680

Blas Arroyo, J. (1999). Las actitudes hacia la variación intradialectal en la sociolingüística hispánica. Estudios Filológicos, 34, 44-72.

Buck, M. (1994). La influencia de la lengua materna en el aprendizaje de lenguas extranjeras. Estudios de Lingüística Aplicada, 19/20, 365-375. https://ela. enallt.unam.mx/index.php/ela/article/view/263/243

Canché Teh, B. F. (2014). Uso, actitudes y aprendizaje del maya en la uimQRoo [tesis de doctorado]. Universidad de Barcelona.

Cantos, P. y Sánchez, A. (2011). El inglés y el español desde una perspectiva cuantitativa y distributiva: Equivalencias y contrastes. Estudios ingleses de la Universidad complutense, 19, 15-44.

Castillo, M. (2006). El estudio de las actitudes en el contexto sociocultural: El caso del mexicano de Cuetzalan. Anales de Antropología, 40(1), 283-317.

Castillo, M. (2007). Mismo mexicano pero diferente idioma: Identidades y actitudes lingüísticas en los maseualmej de Cuetzalan. Instituto Nacional De Antropología e Historia (INAH-UNAM).

Congreso de la República, (8 de febrero de 1994). Ley 115 de febrero 8 de 1994. https://www.mineducacion.gov. co/1621/articles-85906_archivo_pdf.pdf

Congreso de la República (12 de julio de 2013). Ley 1651 de 2013. https://www.mineducacion. gov.co/1759/w3-article-381602.html?_noredirect=1\#: :text=Por $\% 20$ medio $\% 20$ de $\% 201 \mathrm{la} \% 20$ cual,otras $\% 20$ disposiciones $\% 2$ Dley $\% 20 \mathrm{de} \% 20$ biling\%C3\%BCismo

Criado, M. (2004). Percepciones y actitudes en torno a la lengua española en Estados Unidos. Migr. Inter., 2(4). http://www.scielo.org.mx/scielo.php?script=sci_arttext\&pid=S1665-89062004000200005
Dávila, D. (2012). Capital lingüístico en inglés y desigualdad de oportunidades educativas: Exploración en dos colegios oficiales de Bogotá [tesis de grado]. Universidad Nacional de Colombia.

Falcón, P. y Mamani, L. (2017). Actitudes lingüísticas en contextos interculturales: Población asháninka Bajo Chirani. RLA. Revista de Lingüística Teórica y Aplicada, 55(1), 95-115. https://dx.doi.org/10.4067/ S0718-48832017000100095

García, M. y Senovilla, A. (1998). El papel de la lengua materna en la enseñanza-aprendizaje de una gramática para comunicar. Actas del IX Congreso Internacional de ASELE (pp. 301-308). https:// cvc.cervantes.es/ensenanza/biblioteca_ele/asele/ pdf/09/09_0304.pdf

Gómez J. R. (1998). Actitudes lingüísticas en una comunidad bilingüe y multidialectal: Área Metropolitana de Valencia. Cuadernos de Filología, 28. Universitat de València.

Gómez, J. R. (2002). Lenguas en contacto y actitudes lingüísticas en la comunidad de Valencia. En J. Blas, M. Casanova, S. Fortuño y M. Porcar (eds.), Estudios sobre lengua y sociedad. Universitat Jaume.

González, J. (2008). Metodología para el estudio de las actitudes lingüísticas. Actas del XXXVII Simposio Internacional de la Sociedad Española de Lingüística (SEL). https://core.ac.uk/download/pdf/83571986. pdf

Henríquez, M. y Olate, A. (2010). Actitudes lingüísticas de profesores mapuche de Educación Básica: Vigencia y enseñanza del mapudungun en el contexto educativo. Literatura y Lingüística, 22, 103-116. https://scielo.conicyt.cl/scielo.php?script=sci_arttext\&pid=S0716-58112010000200008

Hernández, A. (2012). Actitudes idiomáticas de los estudiantes de la Universidad Nacional de Bogotá ante lenguas indígenas y el español. Portal de lenguas de Colombia, diversidad y contacto. https:// lenguasdecolombia.caroycuervo.gov.co/contenido/ Espanol-de-Colombia/Articulo/contenido/95\&

Hernández-Campoy, J. (2004). El estudio de las actitudes y su medición en sociolingüística. Universidad de Murcia. Tonos digitales: Revista de Estudios Filológicos, 8. https://digitum.um.es/digitum/bitstream/10201/50065/1/El\%20fenomeno\%20de\%20 las\%20actitudes.pdf 
Hernández Campoy, J. M. y Almeida, M. (2005). Metodología de la investigación sociolingüística. Editorial Comares.

Huguet, Á. y Llurda, E. (2001). Language attitudes of school children in two Catalan/Spanish Bilingual Communities. Internacional Jornal of Bilingual Education and Bilingualism, 4, 267-282.

Izquierdo Díaz, J. S. (2013). La actitud del aprendiz de una segunda lengua (L2) hacia su lengua materna (L1) como factor en el proceso de aprendizaje de la L2. Revista Nebrija de Lingüística Aplicada, 13. https:// www.nebrija.com/vida_universitaria/servicios/ pdfpublicaciones/ActasNebrija_PrimerCongreso_ volumn1.pdf

Janés, J. (2006). Las actitudes hacia las lenguas y el aprendizaje lingüístico. Revista Interuniversitaria de Formación del Profesorado, 20(2), 117-132.

Klein, W. (1986). Second language acquisition. Cambridge University Press.

Lasagabaster, D. (2003). Trilingüismo en la enseñanza: Actitudes hacia la lengua minoritaria, la mayoritaria y la extranjera. Editorial Milenio.

Lasagabaster, D. (2004). Un análisis de las actitudes hacia el inglés como lengua extranjera por medio de grupos de discusión. Estudios de Lingüística Aplicada, 40. http://ela.enallt.unam.mx/index.php/ ela/article/viewFile/651/731

López Morales, H. (2004). Sociolingüística. Gredos.

López Morales, H. (2010). Ponencia inaugural: Presente y futuro del español. Memorias XLV Congreso. El Camino de Santiago: Encrucijada de lenguas y culturas.

Lopreto, G. (2018). Hablas en el aula: La diversidad lingüística en el aula [EPub]. Editorial de la Universidad Nacional de La Plata (EDULP). https://libros.unlp. edu.ar/index.php/unlp/catalog/book/996

Malinowski, M. (2000). Interferencias de una segunda lengua en el estudio del español como lengua extranjera, en estudiantes polacos. https://cvc.cervantes.es/ ensenanza/biblioteca_ele/asele/pdf/11/11_0493.pdf

Mamani, E. (2011). Contrapunto de voces en la realidad sociolingüística de Tilcara Jujuy-Argentina [EPub]. uMss-Funproeib Andes-Plural Editores. http://bvirtual.proeibandes.org/bvirtual/docs/pub_estela.pdf

Masuda, K. (2104). Actitudes lingüísticas en torno al E/LE en Japón: Influencia de la actitud monocentrista hacia la LM ante la adquisición de la LE [Trabajo de Maestría]. Universidad de Barcelona.

Matos Aldana, T. S. (2010). Actitudes lingüísticas hacia el francés lengua extranjera: El caso de los estudiantes de las Escuelas de Idiomas Modernos y de Educación mención Lenguas Modernas en la Universidad de los Andes [Tesis de maestría, Universidad de los Andes]. http://www.revistaeducacion.mec.es/re345/ re345.pdf

Minera, L. (2010). La motivación y las actitudes de aprendizaje del E/LE en los estudiantes no hispanistas de la Universidad LMu de Múnich. Revista Nebrija de Lingüística Aplicada, 8. https://www. nebrija.com/revista-linguistica/files/articulosPDF/ articulo_530b5b3b23628.pdf

Ministerio de Educación Nacional (2014). Colombia very well: Programa Nacional de Inglés 2015-2025 [documento de socialización]. https://www.ucn.edu.co/ institucion/sala-prensa/noticias/Documents/2014/ Documento\%20PROGRAMA\%20NACIONAL\%20 DE\%20INGL\%C3\%89S\%202015-2025.pdf

Montoya, A. (2013). La construcción de las actitudes lingüísticas: Exploración en dos colegios bilingües bogotanos [tesis de maestría, Universidad Nacional]. http://bdigital.unal.edu.co/11560/1/angelicamontoyaavila.2013.pdf

Moreno Fernández, F. (1998). Principios de sociolingüística y sociología del lenguaje. Editorial Ariel.

Moreno Fernández, F. (2007). Adquisición de segundas lenguas y sociolingüística. Revista de Educación, 343, 55-70. http://www.revistaeducacion.educacion.es/ re343/re343_03.pdf

Muñoz, M. (2019). Las actitudes y creencias lingüísticas de estudiantes universitarios. RIDE. Revista Iberoamericana para la Investigación y el Desarrollo Educativo, 9(18), 748-767. https://dx.doi. org/10.23913/ride.v9i18.444

Rodríguez, A. (2018). Actitudes e ideologías lingüísticas de docentes de español: Entre la corrección y el valor de la diversidad. Análisis, 50(91). https://revistas. usantotomas.edu.co/index.php/analisis/article/ viewFile/3701/pdf

Trudgill, P. J. (1983). Sociolinguistics: An introduction to language and society. Penguin. 
